ESJ Social Sciences

\title{
Etude exploratoire de l'impact de l'approche processus sur la performance : Cas des entreprises marocaines
}

\author{
Abderrazak Benammi, PhD Student \\ Kacem Taj, PhD Professor \\ Faculté des Sciences Juridiques, Economiques et sociales Ain Chock \\ Université Hassan II, Casablanca, Maroc
}

Doi:10.19044/esj.2021.v17n27p167

Submitted: 09 June 2021

Accepted: 02 August 2021

Published: 31 August 2021
Copyright 2021 Author(s)

Under Creative Commons BY-NC-ND

4.0 OPEN ACCESS

Cite As:

Benammi A. \& Taj K. (2021). Etude exploratoire de l'impact de l'approche processus sur la performance : Cas des entreprises marocaines. European Scientific Journal, ESJ, 17 (27), 167. https://doi.org/10.19044/esj.2021.v17n27p167

\section{Résumé}

La question de l'évaluation de l'impact de l'approche processus sur la performance constitue un domaine d'investigation non encore bien exploré au Maroc. Du point de vue académique, il s'agit d'approfondir les investigations afin d'enrichir les études antérieures portant sur la problématique de l'approche processus et la performance des organisations. Du point de vue management, il s'agit de satisfaire un besoin des entreprises en apportant des éléments de réponses à la question de contribution de l'approche processus à l'amélioration de la performance. En se basant sur le modèle «Business Process Management Maturity » et «Balanced Score Card », une étude qualitative exploratoire a été menée avec dix-sept professionnels (dont trois experts) opérants dans des secteurs d'activités différents, afin de comprendre la possibilité d'application de la recherche notamment l'existence et les insuffisances des construits sur le terrain. Cette étude exploratoire nous a permis d'examiner les facteurs clés de succès de l'approche processus et leur impact sur la performance de l'entreprise marocaine en proposant à la fin un modèle de recherche reliant l'approche processus et la performance. Cette recherche s'est confrontée, néanmoins, à des limites liées à l'étroitesse de l'échantillon et à l'impossibilité d'accéder aux données. Cette étude ouvre de nouvelles perspectives en termes de recherche comme le test du modèle proposé dans divers secteurs afin d'avoir une validité externe. 
Mots clés : Performance, approche processus, Modèle « Business Process Management Maturity », tableau de bord prospectif, modèle

\title{
Exploratory study of the Impact of the Process Approach on Performance: A Case of Moroccan Companies
}

\author{
Abderrazak Benammi, PhD Student \\ Kacem Taj, PhD Professor \\ Faculté des Sciences Juridiques, Economiques et sociales Ain Chock \\ Université Hassan II, Casablanca, Maroc
}

\begin{abstract}
The evaluation of the impact of the process approach on performance is an area of investigation that has not yet been well explored in Morocco. From an academic point of view, it is a question of deepening the investigations in order to enrich previous studies on the problems of the process approach and the performance of organizations. From a management point of view, it is about satisfying a business need by providing some answers to the question on how the process approach contributes to improving performance. Based on the "Business Process Management Maturity" and "Balanced Score Card" model, an exploratory qualitative study was carried out with seventeen professionals (including three experts) operating in different business sectors. This is aimed at understanding the possible application of research and, in particular, the existence and the inadequacies of constructions in the field. This exploratory study help to examine the key success factors of the process approach and their impact on Moroccan company performance by proposing at the end a research model a correlation between the process approach and performance. This research, however, faced limitations related to the small size of the sample and the inability to access the data. This study opens up new perspectives in terms of research such as testing the proposed model in various sectors to obtain external validity.
\end{abstract}

Keywords: Performance, process approach, Business Process Management Maturity Model, Balanced Score Card, model

\section{Introduction}

Le marché et l'environnement économique connaissent une évolution caractérisée par un durcissement de la compétitivité économique et par une personnalisation et diversification accrues des produits (Franchini et al., 1997). Par conséquent, les entreprises sont appelées à se fixer des objectifs élevés en termes de qualité, de flexibilité, de rapidité de développement de 
nouveaux produits (innovation) et de leur mise sur le marché. Cela sollicite une capacité d'innover et une maîtrise des changements organisationnels et technologiques. Ansari (1999), affirme que la pérennité de l'entreprise est liée à des transformations que doivent connaitre les structures de l'organisation et les pratiques managériales dans la recherche d'une optimisation durable de la production. Dans le même ordre d'idées, David et al. (1999), soulignent que le monde de la production a totalement changé (l'obligation de la qualité et de différentiation des produits, le respect des délais et des coûts). Par conséquent, au cours de ces dernières années, de nouvelles approches d'organisation et modes de management ont été développés et proposés contribuant à répondre à de nouveaux objectifs de production et permettant la maîtrise de la performance.

Si l'objectif des entreprises est la réalisation de la performance et indirectement l'atteinte des résultats, les normes ISO 9000 sont capables de répondre à ces attentes. Parmi les caractéristiques des normes ISO 9000, il s'agit d'agir sur les échanges horizontaux et de favoriser les liens de communication internes notamment avec l'approche transversale des processus qui constitue une des plus importantes exigences de ces normes. Les normes permettent à l'entreprise d'être vue sous l'angle d'une vision des processus inter reliés qui communiquent entre eux. Malgré ses véritables apports, ces normes ne constituent pas la réponse totale aux attentes des entreprises, ni un moyen suffisant qui mène vers l'excellence, sachant que des entreprises cherchent uniquement la certification pour des besoins commerciaux. Devant cette situation, le concept de l'approche processus (Business Process Management (BPM)) apparaît comme un mode de management susceptible de maintenir et d'améliorer la performance des entreprises et soutenir leurs capacités concurrentielles.

Ainsi, de nombreuses entreprises ont commencé en interne à améliorer continuellement leurs processus sous différents aspects pour répondre aux changements d'environnement et aux besoins des clients (Siha \& Saad, 2008). Par conséquent, l'approche processus est devenue le mode de management commercial et technologique le plus populaire ces dernières années (Garimella, Lees \& Williams, 2008). En fait, l'approche processus n'est pas une technologie mais une discipline de gestion orientée processus (Hill et al., 2008). Elle est une combinaison de mesures, des outils et des technologies pour concevoir et régir les processus (Garimella, Lees \& Williams, 2008). L'approche processus est une approche efficace pour gouverner et améliorer les activités fondamentales des opérations d'une entreprise telles que le développement de produits, le marketing, la coordination entre fonctions et d'autres éléments majeurs (Zairi, 1997).

En matière de performance, une large gamme de possibilités s'ouvre. Neely et al. (2005) montrent la difficulté de traiter ce construit en raison de 
diverses terminologies et façons de l'examiner. La difficulté réside dans le caractère polysémique du construit de la performance qui, jusqu'à ce jour, les chercheurs n'arrivent pas à délimiter les frontières ou à proposer une définition unique qui fait l'unanimité. En outre, de nombreuses variables influent sur la performance, allant de l'alignement stratégique, la gouvernance, les méthodes, la technologie, les personnes et la culture (De Bruin, 2009) à la gestion des actifs incorporels (Chareonsuk \& Chansa-ngavej, 2010). De plus, on peut traiter la performance au niveau opérationnel et organisationnel ou dans différentes dimensions (Rowe \& Morrow, 1999). Une littérature abondante sur l'approche processus affirme que les organisations pourraient améliorer leur performance globale en adoptant une vision processus de l'entreprise. Cependant, il y a un manque de recherche empirique dans ce domaine (Škrinjar et al., 2008). Alors que la littérature présente plus qu'une abondance d'avantages liés à l'approche processus, leur confirmation empirique reste rare (Sharma, 2005).

S'inscrivant dans cette logique, Il nous a semblé utile et intéressant d'engager un travail de recherche autour de cette thématique d'évaluation de la performance, dans un contexte marocain, afin de répondre à une interrogation principale qui fait l'objet de notre problématique à savoir :

Quel est l'impact de l'adoption de l'approche processus sur la performance globale des entreprises marocaines?

\section{Cadre théorique de la Recherche}

Dans le but de répondre à notre problématique nous avons opté pour un positionnement théorique de causes à effets (De Vaujany, 2009). Il s'agit de montrer un lien statistique significatif entre l'approche processus et la performance qu'elle engendre.

Pour cette étude, nous avons pris le pari de proposer un modèle de recherche issue de deux modèles : Business Process Management Maturity (BPMM), de De Bruin Tonia et Rosemann Michael (2005) et le Balanced Score Card (BSC) de Kaplan et Norton (1992).

Le BPMM suggère que l'approche processus aboutit à des processus réussis permettant la réalisation de la performance grâce aux six facteurs clés de succès suivants : l'alignement stratégique, la gouvernance, les méthodes, les ressources humaines, la technologie de l'information et la culture.

Ces facteurs sont fortement cités dans la littérature tel que présenté dans le tableau suivant : 
Tableau 1. Les six éléments fondamentaux de l'approche processus dans la littérature (Source : adapté de M. Rosemann and J. vom Brocke, 2010, P 114)

\begin{tabular}{|l|l|}
\hline \multicolumn{1}{|c|}{ Variables } & \multicolumn{1}{|c|}{ Source initiale } \\
\hline $\begin{array}{l}\text { Alignement } \\
\text { stratégique }\end{array}$ & $\begin{array}{l}\text { Elzinga et al., 1995; Hammer, 2001; Hung, 2006; Jarrar, Al- } \\
\text { Mudimigh et Zairi, 2000; Pritchard et Armistead, 1999; Puah K.Y. et } \\
\text { Tang K.H, 2000; Zairi, 1997; Zairi et Sinclair, 1995 }\end{array}$ \\
\hline Gouvernance & $\begin{array}{l}\text { Braganza et Lambert, 2000; Gulledge et Sommer, 2002; Harmon, } \\
\text { 2005; Jarrar, Al-Mudimigh et Zairi, 2000; Pritchard et Armistead, } \\
1999\end{array}$ \\
\hline Méthodes & $\begin{array}{l}\text { Adesola et Baines, 2005; Harrington, 1991; Kettinger et al., 1997; } \\
\text { Pritchard et Armistead, 1999; Zairi, 1997 }\end{array}$ \\
\hline $\begin{array}{l}\text { Technologie de } \\
\text { l'information }\end{array}$ & $\begin{array}{l}\text { McDaniel, 2001; Gulledge et Sommer, 2002; Hammer et Champy, } \\
1993\end{array}$ \\
\hline $\begin{array}{l}\text { Ressources } \\
\text { humaines }\end{array}$ & $\begin{array}{l}\text { Elzinga et al., 1995; Llewellyn et Armistead, 2000; Hung, 2006; } \\
\text { Pritchard et Armistead, 1999; Zairi, 1997; Zairi et Sinclair, 1995 }\end{array}$ \\
\hline Culture & $\begin{array}{l}\text { Elzinga et al., 1995; Llewellyn et Armistead, 2000; Pritchard et } \\
\text { Armistead, 1999; Spanyi, 2003; Zairi, 1997; Zairi et Sinclair, 1995 }\end{array}$ \\
\hline
\end{tabular}

Le BSC considère la performance comme un concept multidimensionnel subdivisé en quatre axes : axe client, axe financier, axe processus interne et axe innovation et apprentissage organisationnel.

Une revue de littérature des deux concepts clés de notre problématique est entamée dans une première étape, afin de répondre à notre question principale de recherche dans une seconde étape.

\section{Approche processus}

L'approche processus, en tant que méthode d'organisation, de management ou de consulting, est née dans la qualité au début des années 90 et utilisée dans le milieu informatique pour modéliser et analyser une activité dans le but de l'informatiser. Elle a été utilisée à l'époque comme méthode de sauvetage des entreprises en difficulté en les reconstruisant de zéro grâce au « Business Process Reengineering » (BPR). L'approche processus est remise sur le devant de la scène et est devenu un outil de management, grâce auquel, l'organisation devient maîtrisée puisqu'elle a un lien direct avec le produit ou le service. Un processus n'a pas d'existence s'il n'a pas comme résultat un produit ou un service destiné à un ou des clients internes ou externes identifiés. La mise en œuvre de l'approche processus constitue une occasion pour démanteler les barrières au sein de l'entreprise. En fait, un processus efficace est un processus où les interfaces entre les fonctions auxquelles il fait appel ne constituent pas des causes de conflits mais, au contraire, une occasion pour réaliser des progrès et des améliorations.

La version 2000 de la norme ISO 9001 prévoit l'utilisation de l'approche processus comme outil de management. Cela consiste à identifier et décrire les processus nécessaires à la réalisation des produits et assurer 
ensuite le bon fonctionnement et l'amélioration continue de chaque processus. L'approche conduit donc à abandonner la logique hiérarchique basée sur les spécialités et les métiers au profit d'un équilibre entre la logique hiérarchique et une logique transversale.

L'objectif de cette approche est de fournir un produit de bonne qualité pour le client interne et externe au moindre coût pour l'organisme.

De nombreux chercheurs ont tenté de présenter une définition de

l'approche processus tel que présenté dans le Tableau 2 suivant :

Tableau 2. Définitions de l'approche processus proposées par la littérature

\begin{tabular}{|c|c|}
\hline Auteur & Définition \\
\hline Elzinga et al., 1995 & $\begin{array}{l}\text { Une approche systématique et structurée permettant d'analyser, améliorer, } \\
\text { contrôler et gérer les processus dans le but d'améliorer la qualité des produits } \\
\text { et prestations de service. }\end{array}$ \\
\hline Zairi, 1997 & $\begin{array}{l}\text { Une approche structurée pour analyser et améliorer continuellement les } \\
\text { activités fondamentales telles que la fabrication, la commercialisation, la } \\
\text { communication et autres éléments majeurs des opérations d'une } \\
\text { entreprise (...) l'approche processus concerne les principaux aspects des } \\
\text { opérations commerciales où il y a fort effet de levier et une grande proportion } \\
\text { de la valeur ajoutée. }\end{array}$ \\
\hline Lee et Dale, 1998 & $\begin{array}{l}\text { Une approche axée sur le client pour gérer et améliorer tous les processus } \\
\text { dans une entreprise. }\end{array}$ \\
\hline Jarrar et al., 2000 & $\begin{array}{l}\text { L'approche processus devrait se concentrer sur l'amélioration des processus } \\
\text { dans la chaîne d'approvisionnement ainsi que la gestion du changement, la } \\
\text { gestion et le développement des personnes. }\end{array}$ \\
\hline $\begin{array}{l}\text { Van der Aalst et al., } \\
2003\end{array}$ & $\begin{array}{l}\text { L'approche processus vise à atteindre la création de la valeur optimisée par } \\
\text { le contrôle et l'analyse des processus opérationnels par l'appui des techniques } \\
\text { et des outils logiciels. }\end{array}$ \\
\hline $\begin{array}{l}\text { Hans Brandenburg, } \\
\text { Jean-Pierre Wojtyna, } \\
2003\end{array}$ & $\begin{array}{l}\text { Une méthode d'analyse ou de modélisation. Elle consiste à décrire de façon } \\
\text { méthodique une organisation ou une activité, généralement dans le but d'agir } \\
\text { dessus. }\end{array}$ \\
\hline Michel Cattan, 2008 & $\begin{array}{l}\text { L'approche processus consiste à considérer l'organisme comme un ensemble } \\
\text { de petits organismes qui contribuent à réaliser un même produit ou à rendre } \\
\text { un même service. Il en résulte que, dans une telle approche, le personnel et } \\
\text { l'encadrement doivent se comporter comme des entrepreneurs sur un marché } \\
\text { concurrentiel. Là encore, rien de bien nouveau si l'on se réfère à la méthode } \\
\text { dite RCFI (Relation Client-Fournisseur Internes), outil que les qualiticiens } \\
\text { connaissent bien pour l'avoir utilisé depuis de nombreuses années. }\end{array}$ \\
\hline $\begin{array}{lcc}\text { Norme } & \text { ISO } & 9004 \\
\text { version } 2000 & \end{array}$ & $\begin{array}{l}\text { L'approche processus désigne l'application d'un système de processus au } \\
\text { sein d'un organisme, ainsi que l'identification, les interactions et le } \\
\text { management de ces processus. L'un des avantages de l'approche processus } \\
\text { est la maîtrise permanente qu'elle permet des relations entre les processus au } \\
\text { sein du système de processus, ainsi que leurs combinaisons et interactions }\end{array}$ \\
\hline
\end{tabular}

En se référant au BPMM, le succès de l'approche processus dépend de six éléments fondamentaux suivants : l'alignement stratégique, la gouvernance, les méthodes, les ressources humaines, la technologie de 
l'information et la culture (Rosemann \& Brocke, 2010).

Nous présentons ci-dessous une synthèse de revue de littérature de ces facteurs :

L'Alignement stratégique : elle est définie comme étant l'étroite liaison entre les priorités organisationnelles et les processus d'entreprise permettant une action efficace et continue pour améliorer les performances de l'entreprise.

La gouvernance : elle établit une responsabilité appropriée et transparente en termes de rôles et de responsabilités pour les différents niveaux de l'approche processus (portefeuille, programme, projet et opérations).

Les méthodes : elles sont définies comme l'ensemble d'outils et de techniques qui prennent en charge et guident les activités tout au long du cycle de vie du processus.

Les technologies de l'information : les solutions informatiques permettant l'analyse et la modélisation des processus se manifestent de plus en plus sous la forme de systèmes d'information dédiés aux processus (Dumas et al., 2005).

Le personnel (ressources humaines) : le personnel est défini comme étant des individus et des groupes qui améliorent et appliquent continuellement leurs compétences et leurs connaissances en matière de processus et leur gestion.

La culture : elle intègre les valeurs et les croyances collectives en ce qui concerne l'organisation centrée sur les processus. Les études de cas comparatives démontrent clairement le fort impact de la culture sur le succès de l'approche processus (De Bruin, 2009).

Comme précisé ci-dessus, le BPMM suggère que l'approche processus permette à l'entreprise de se doter des processus performants ou réussis qui conduisent à l'amélioration de la performance globale. Le concept "succès de processus" est considéré comme une variable médiatrice entre l'approche processus et la performance.

\section{Performance variable dépendante dans la recherche en approche processus}

Un nombre important de chercheurs étudient, depuis plusieurs années, les liens entre des variables contextuelles comme la stratégie, la structure, la qualité totale, etc. et leurs effets sur la performance de l'entreprise. Ces études s'intéressent la plupart du temps à la performance sous son angle financier. Or, comme précisé par Kaplan et Norton (1998) l'entreprise travaille dans un environnement de plus en plus complexe et l'utilisation unique du volet financier de la performance ne suffit plus. Pour ces auteurs, la performance est devenue multicritères et sa mesure doit tenir compte de cette particularité. Les travaux de Kaplan et Norton (1998), portent sur ce sujet et présentent un 
modèle de mesure de la performance, appelé Balanced scorecard (BSC), qui tient compte à la fois du volet financier et intègre des volets liés aux opportunités de croissance de l'entreprise, telles que les clients, les processus internes, l'apprentissage organisationnel et l'innovation. En revanche, plusieurs travaux empiriques cherchent à délimiter le champ de la performance puisque la problématique de définition et d'évaluation de ce concept persiste encore dans le domaine du management.

Le modèle de Kaplan et Norton permet de regrouper les indicateurs de performance en quatre grandes classes (appelés visions ou axes) qui sont les suivant :

- La vision client : permet de mesurer la performance concernant la satisfaction et fidélité des clients et d'avoir une idée claire sur l'identité des segments cibles de marché (Selmer, 2003).

- La vision financière: sert de fil conducteur aux objectifs de l'entreprise et aux indicateurs des autres axes. Chaque indicateur sélectionné doit faire partie d'une chaîne de relations de causes à effet dont le but ultime est d'améliorer la performance financière.

- Vision processus interne : mesure la capacité de l'entreprise à maîtriser ses modes de fonctionnement. C'est-à-dire, elle reflète la manière avec laquelle l'entreprise transforme les éléments d'entrée en éléments de sortie de la façon la plus optimale possible.

- Vision innovation et apprentissage organisationnel : mesure l'habileté pour une entreprise d'innover, d'améliorer et d'apprendre. C'est un axe ou l'entreprise doit exceller pour améliorer sa performance.

Le tableau suivant présente les principaux axes de la performance selon le BSC et quelques exemples d'indicateurs.

Tableau 3. Les axes de la performance selon Kaplan et Norton (1992) (Source : Helène (2000))

\begin{tabular}{|c|c|c|}
\hline Axe & $\begin{array}{c}\text { Déterminants de la } \\
\text { performance }\end{array}$ & Exemple d'indicateurs \\
\hline Financier & $\begin{array}{l}\text { - Accroissement du chiffre } \\
\text { d'affaire. } \\
\text { - Réduction des coûts et } \\
\text { amélioration de la } \\
\text { productivité. } \\
\text { - Utilisation de l'actif. } \\
\text { - Réduction du risque. }\end{array}$ & $\begin{array}{l}\text { - Croissance des ventes. } \\
\text { - } \% \text { de bénéfice net. } \\
\text { - Rendement sur capital } \\
\text { investi. } \\
\text { - Coûts unitaires. }\end{array}$ \\
\hline Clients & 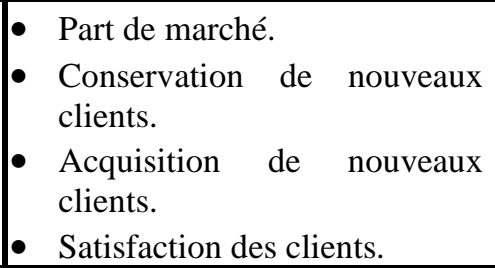 & $\begin{array}{l}\text { - \% des ventes réalisées auprès } \\
\text { des clients existants. } \\
\text { - \% des ventes réalisées auprès } \\
\text { de nouveaux clients. } \\
\text { - Degré de satisfaction des } \\
\text { clients. }\end{array}$ \\
\hline
\end{tabular}




\begin{tabular}{|c|c|c|}
\hline & Rentabilité par segment. & - Taux de retour des produits. \\
\hline $\begin{array}{l}\text { Processus } \\
\text { Interne }\end{array}$ & $\begin{array}{l}\text { Qualité, réactivité, } \\
\text { productivité, coût pour chacun } \\
\text { des grands processus d'une } \\
\text { entreprise soit : } \\
\text { - L'innovation, } \\
\text { - La production, } \\
\text { - Le service après-vente. }\end{array}$ & $\begin{array}{l}\text { - Argent investi en recherche } \\
\text { et développement. } \\
\text { - \% des ventes réalisées avec } \\
\text { des nouveaux produits. } \\
\text { - Temps de réponse aux appels } \\
\text { de service. } \\
\text { - Coûts standard. }\end{array}$ \\
\hline $\begin{array}{l}\text { Innovation et } \\
\text { Apprentissage } \\
\text { Organisationnel }\end{array}$ & $\begin{array}{l}\text { - Le potentiel des salariés. } \\
\text { - Réorientation } \\
\text { compétences. } \\
\text { - Capacités des } \\
\text { d'information. } \\
\text { - Alignement des } \\
\text { individuels aves objectifs } \\
\text { l'entreprise. }\end{array}$ & $\begin{array}{l}\text { - Taux de satisfaction des } \\
\text { employés. } \\
\text { - Argent investi en formation. } \\
\text { - Disponibilité } \\
\text { l'information. } \\
\text { - Nombre de suggestions par } \\
\text { employé. }\end{array}$ \\
\hline
\end{tabular}

Les entreprises cherchent à disposer d'un système de mesure de la performance multidimensionnel pour relever le défi de la compétitivité. Le cadre d'analyse du (BSC) apparaît suffisamment complet pour servir de base pour évaluer les systèmes de mesure de la performance existants et proposer des pistes d'améliorations qui permettront d'accroître la performance des entreprises. Le (BSC) est utilisé dans notre modèle pour expliquer la variable dépendante «performance ».

\section{Approche processus et performance}

Plusieurs entreprises se sont investies dans une démarche de gestion par les processus dans la recherche de l'amélioration de la qualité de leurs produits et services. Ce recours vers l'approche processus est justifié par la satisfaction des parties prenantes (clients, employés, actionnaires, etc.). La satisfaction de ces derniers permet à l'entreprise d'acquérir un avantage concurrentiel. De plus, les chefs d'entreprise croient fortement à une relation positive entre l'approche processus et la performance. En fait, il existe déjà des travaux sur l'évaluation de la performance dans une logique d'approche processus. Les résultats des investigations traitant cette thématique semblent convergeant vers une relation positive entre ces deux concepts. Une revue de la littérature est déployée afin de présenter les études ayant étudiée l'effet de l'approche processus sur la performance de l'entreprise [Davenport (1993), Hammer et Champy (1993), Ginn et Barlog (1994), Day (1994), Elzinga et al. (1995), Garvin (1995), Hinterhuber (1995), Fitzgerald et Murphy (1996), Kaplan et Norton (1996), DeToro et McCabe (1997), Ittner et Larcker (1997), Zairi (1997), Frei, Kalakota, Leone et Marx (1999), Pritchard et Armistead (1999), McCormack (1999), Llewellyn et Armistead (2000), Hammer (2001), McCormack (2001a), McCormack et Johnson (2001) ... etc]. 
À l'époque de l'émergence de l'approche processus en tant que discipline au milieu des années 1990, après l'introduction de la réingénierie des processus de Hammer et Champy (1993) et l'innovation de processus de Davenport (1993), l'intérêt pour l'approche processus est justifié par :

$\checkmark$ Besoin d'améliorer la réactivité et la qualité et de gérer les risques concurrentiels (Pritchard \& Armistead, 1999);

$\checkmark$ Mondialisation, évolution de la technologie, réglementation, action des parties prenantes et démantèlement des frontières commerciales (Armistead, 1996) ;

$\checkmark$ Compétitivité de l'industrie sur le marché international (Elzinga et al., 1995).

Depuis lors, l'investissement et l'intérêt pour l'amélioration et la gestion des processus de l'entreprise se sont poursuivis. Pendant ce temps, Gartner (2009) a considéré l'amélioration des processus comme la priorité commerciale numéro un des chefs d'entreprises. L'approche processus permet à l'entreprise d'atteindre des avantages lui permettant une compétitivité accrue et la réalisation de la performance globale à travers :

$\checkmark \mathrm{La}$ réduction des temps de cycle, l'utilisation des nouvelles technologies de l'information et l'obtention du contrôle managérial (Gulledge \& Sommer, 2002; Zairi, 1997);

$\checkmark$ La réduction des coûts, des ressources et / ou des frais généraux (Hammer, 2001 ; Zairi, 1997) ;

$\checkmark$ Une augmentation de la satisfaction des clients et une meilleure capacité de répondre aux besoins de ces clients (Hammer, 2001 ; Pritchard \& Armistead, 1999 ; Zairi, 1997 ; DeToro \& McCabe, 1997) ;

$\checkmark$ Une réduction du délai de mise sur le marché et une amélioration de la prestation de services (Gulledge \& Sommer, 2002 ; Zairi, 1997) et de la qualité (Elzinga et al., 1995 ; Hammer, 2001 ; Zairi, 1997) ;

$\checkmark$ La gestion du changement et les problèmes culturels (DeToro \& McCabe, 1997; Llewellyn \& Armistead, 2000; Pritchard \& Armistead, 1999) ;

$\checkmark$ Un meilleur travail d'équipe (Zairi, 1997);

$\checkmark$ L'augmentation du niveau d'autonomisation des employés entraîne une réduction des barrières transversales (DeToro \& McCabe, 1997) ;

$\checkmark$ L'optimisation de toutes les étapes de la chaîne de valeur conduisant à une productivité accrue (Hinterhuber, 1995) ;

$\checkmark$ Augmentation de la valeur ajoutée en se traitant les activités noncompétitives et en se concentrant sur les compétences de base (Hinterhuber, 1995). 
Des études empiriques ont été menées testant la relation entre l'approche processus et la performance, ces études montrent des relations positives entre ces deux concepts (voir Tableau 4).

Tableau 4. Etudes empiriques de la relation : Approche processus - performance

\begin{tabular}{|l|l|ll|}
\hline \multicolumn{1}{|c|}{ Auteur } & \multicolumn{1}{c|}{$\begin{array}{c}\text { Variables } \\
\text { indépendantes }\end{array}$} & \multicolumn{3}{|c|}{ Variables dépendantes } & \\
\hline Kohlbacher (2009) & Approche processus & Performance non financière & \\
\hline $\begin{array}{l}\text { Kohlbacher et al. } \\
(2011)\end{array}$ & $\begin{array}{l}\text { Culture «approche } \\
\text { processus » }\end{array}$ & $\begin{array}{l}\text { Performance financière, délai de } \\
\text { livraison, fiabilité des livraisons }\end{array}$ \\
\hline Zaheer et al. (2010) & Approche processus & $\begin{array}{l}\text { Performance des employés et de } \\
\text { l'organisation }\end{array}$ \\
\hline $\begin{array}{l}\text { Kohlbacher et } \\
\text { Reijers (2013) }\end{array}$ & Approche processus & Performance organisationnelle & \\
\hline
\end{tabular}

Nous rappelons que la performance reste un concept multidimensionnel (financier, organisationnel, structurel, etc.) dont la définition ne fait pas l'unanimité des scientifiques. Une des définitions proposées tende vers l'intégration de l'ensemble des parties prenantes telles que les actionnaires, les employés, les clients, les fournisseurs, etc. Les études existantes dans la littérature montrent que la performance peut être évaluée de diverses angles qui peuvent être larges ou restreintes. Cela dépend de certaines variables tel que le contexte de l'étude et les contraintes de la recherche.

\section{Cadre empirique de la recherche}

Notre objectif à travers cette étude vise à examiner les facteurs clés de succès de l'approche processus et d'étudier leurs impacts sur la performance de l'entreprise. Pour ce faire, nous avons adopté une approche qualitative (étude exploratoire) afin de rechercher les préalables et les causes d'un phénomène (la performance) par l'exploration des facteurs (approche processus) qui entrent en jeu tout au long d'un processus (Bompar, 2010). Il s'agit d'une stratégie de recherche qui est reconnue par les scientifiques et qui est largement utilisée dans les sciences de gestion, et est considérée comme l'une des méthodes appropriées pour produire de la connaissance valide.

Notre choix de procéder à une étude exploratoire a pour objectif d'expliquer et de découvrir un phénomène peu étudié dans le contexte des pays émergeant comme le Maroc. En effet, à notre connaissance, l'implémentation de l'approche processus et la vérification de son effet sur la performance n'ont pas fait suffisamment l'objet, d'études théoriques et empiriques.

\section{L'échantillonnage et le souci de la représentativité}

Le choix de l'échantillon dans les recherches de types qualitatives exige une véritable rigueur de la part du chercheur. En effet, ce choix doit être basé sur la précision et non pas sur le hasard (Evrard et al., 2003). 
Afin de constituer notre échantillon, nous avons eu recours au ciblage des responsables qui répondent mieux aux exigences et à la finalité de notre recherche, afin que nous puissions tirer d'eux le maximum d'informations.

Nous adoptons les recommandations de Miles et Huberman (1991) qui propose d'arrêter les entretiens, dans le cadre d'une étude empirique, dès lors qu'on estime avoir obtenu le maximum des données nécessaires à la recherche et atteindre ainsi la saturation théorique, qui signifie que les nouvelles données issues des entretiens supplémentaires n'ajoutent plus à la compréhension du phénomène (Gautier, 2009). Cela est conditionné par la satisfaction des critères de la validation de l'étude qualitative: l'acceptation interne, la complétude, la saturation, la cohérence interne et la confirmation externe.

Nous présentons dans ce qui suit le déroulement de l'étude qualitative et ses objectifs, le terrain d'investigation, les méthodes de collecte de données et les résultats de cette étude. L'analyse du contenu est la méthodologie d'analyse retenue pour exploiter les données collectées.

\section{La collecte des données de l'étude exploratoire}

Le chercheur dispose d'un outil principal pour effectuer son enquête exploratoire qualitative. Il s'agit de l'utilisation de l'entretien. Dans la théorie et en pratique, il existe plusieurs types d'entretiens qui varient selon la nature de la recherche et l'objectif recherché. L'entretien peut aussi, en fonction du nombre des répondants, être un entretien individuel ou un entretien collectif (de groupe).

Dans le cadre de cette recherche, nous avons adopté la typologie d'entretien développée par Wacheux (1996) qui propose trois sortes d'entretiens : directifs, non-directifs et semi-directifs. Nous avons mené une série d'entretiens qui concernait deux types d'interlocuteurs. Les premiers entretiens, de type semi-directif, sont effectués avec des acteurs qui constituent nos unités d'analyse (responsables d'entreprises de différents secteurs, responsables qualité, responsables de production, etc.), les seconds entretiens, de type, non directif, appelés aussi «entretiens d'experts ou universitaires » sont dédiés à des personnes qui, par leurs spécialités ou leurs expériences, détiennent de l'expertise permettant de mieux comprendre le problème posé, ses aspects et ses points critiques à prendre en compte (Evrard et al., 2003).

La méthode de l'entretien semi-directif est choisie car elle est considérée comme la plus adaptée pour mieux comprendre les caractéristiques de notre problématique.

Pour étudier l'impact de l'approche processus sur la performance de l'entreprise, un guide d'entretien a été conçu afin de recueillir l'information recherchée (annexe 1). Ce guide nous a permis de poser les mêmes questions à tous les interlocuteurs et nous a servi comme un aide-mémoire en cas d'oubli 
de certains volets à analyser. Ce guide a été conçu autour de quatre thèmes en suivant le fil directeur de notre recherche.

Le guide $d$ 'entretien se compose des thèmes suivants :

- Thème 1 : Comprendre le contexte stratégique de déclenchement d'une démarche d'approche processus ;

- Thème 2: Comprendre les facteurs clés de succès de l'approche processus ;

- Thème 3 : Comprendre les axes de la performance ;

- Thème 4: Comprendre la relation entre l'approche processus et la performance.

Les différents entretiens menés pendant cette phase constituent une source importante d'informations au regard de la richesse des données collectées, auxquelles s'ajoutent les données secondaires (documents, rapports, articles...) qui représentent eux aussi une source considérable d'informations.

\section{Déroulement de l'entretien}

Pour s'informer sur l'impact de l'approche processus sur la performance d'une entreprise, nous nous sommes recourus à l'entretien semi directif pour pouvoir créer une ambiance de sympathie et de compréhension avec les interlocuteurs sans les influencer.

En définitive, nous avons réalisé 17 entretiens en respectant les deux catégories des répondants :

Catégorie I : Directeur d'entreprise, responsables qualité, responsables logistique et manager de projets.

Catégorie II : Experts (consultants).

Nous avons mené 14 entretiens avec des répondants de la catégorie I de type semi-directif.

Concernant les entretiens non-directifs, qui sont en nombre de 3, ils sont effectués avec la catégorie II (voir Tableau 5). Notre démarche consistait à laisser aux experts le champ libre pour s'exprimer de façon plus approfondie, afin d'obtenir le maximum d'informations. Les répondants se présentent dans le tableau suivant : 
Tableau 5. L'échantillon exploratoire de notre étude

\begin{tabular}{|c|c|c|c|c|}
\hline $\mathbf{N}^{\circ}$ & Poste & Secteur & $\begin{array}{ll}\text { Durée } & \text { de } \\
\text { l'entretien }\end{array}$ & Code \\
\hline \multicolumn{5}{|c|}{ Catégorie 1} \\
\hline 1 & Directeur général & Industrie & $1 \mathrm{~h} 40$ & Rép-1 \\
\hline 2 & Responsable qualité & Industrie & $1 \mathrm{~h} 49$ & Rép-2 \\
\hline 3 & Responsable qualité & Industrie & $2 \mathrm{~h} 36$ & Rép-3 \\
\hline 4 & Responsable qualité & Services & $2 \mathrm{~h} 07$ & Rép-4 \\
\hline 5 & Responsable qualité & Industrie & $1 \mathrm{~h} 30$ & Rép-5 \\
\hline 6 & Responsable qualité & Industrie & $1 \mathrm{~h} 56$ & Rép-6 \\
\hline 7 & Responsable qualité & Industrie & $1 \mathrm{~h} 40$ & Rép-7 \\
\hline 8 & Responsable qualité & Industrie & $1 \mathrm{~h} 51$ & Rép-8 \\
\hline 9 & Responsable qualité & Industrie & $1 \mathrm{~h} 48$ & Rép-9 \\
\hline 10 & $\begin{array}{ll}\begin{array}{l}\text { Responsable } \\
\text { qualité/contrôle qualité }\end{array} & \text { assurance } \\
\end{array}$ & Industrie & $1 \mathrm{~h} 35$ & Rép-10 \\
\hline 11 & Responsable plateforme logistique & Industrie & $2 \mathrm{~h} 33$ & Rép-11 \\
\hline 12 & Responsable logistique & Industrie & $2 \mathrm{~h} 50$ & Rép-12 \\
\hline 13 & Responsable qualité & Industrie & $2 \mathrm{~h} 40$ & Rép-13 \\
\hline 14 & Manager de projets & Industrie & $1 \mathrm{~h} 35$ & Rép-14 \\
\hline \multicolumn{5}{|c|}{ Catégorie 2} \\
\hline 15 & Directeur général & Consulting & $1 \mathrm{~h} 35$ & Exp-1 \\
\hline 16 & Directeur général & Consulting & $2 \mathrm{~h} 01$ & Exp-2 \\
\hline 17 & Directeur général & Consulting & $2 \mathrm{~h} 02$ & Exp-3 \\
\hline
\end{tabular}

\section{Méthode d'analyse de contenu}

Les recherches qualitatives s'appuient sur différentes méthodes pour l'analyse et le traitement des données. Elles dépendent de plusieurs variables " les questions de recherche et les résultats attendus (causalité, comportement, représentation, description, test)»(Wacheux, 1996). Dans le cadre de notre recherche nous avons choisi de poursuivre la méthode préconisée par (Thietart et al., 2007), «l'analyse thématique » que nous avons jugée comme la méthode la plus appropriée pour notre recherche. L'analyse de contenu repose, Selon Allard-Poesi et al. (1999), sur «le postulat que la répétition d'unités d'analyse de discours (mots, expressions ou significations similaires, phrases, paragraphes) révèle les centres d'intérêt et les préoccupations des auteurs du discours ».

D'une façon générale, le choix de l'analyse du discours repose sur la technique textuelle de type thématique. Cette analyse est le résultat des données primaires issues de la retranscription des discours liés à l'impact de l'approche processus sur la performance de l'entreprise au Maroc. Nous avons opté pour une approche de type « analyse de contenu » comme modalité de traitement et d'analyse des données recueillies. Celle-ci consiste, comme précisé par Evrard et al. (2003) : 
- A retranscrire les discours issus des données qualitatives recueillies ;

- A découper le texte en unités d'analyse de base ;

- A regrouper ces unités en catégories homogènes, exhaustives et exclusives ;

- A comptabiliser leur fréquence d'apparition selon des règles préétablies.

Au terme de cette phase, une analyse des données est menée nous permettant de dégager des conclusions et nous autorisant a entamé l'étape d'interprétation afin d'affecter les verbatim aux réponses de la question de la recherche.

L'objectif de la phase exploratoire de notre recherche est de mieux connaitre le terrain d'investigation empirique, de s'assurer de la pertinence des hypothèses formulées issues de la revue de littérature. Nous présentons dans ce qui suit les résultats et leur discussion.

\section{Discussion des résultats}

Nous remarquons dans notre échantillon que les répondants appartiennent principalement à l'industrie et les services ce qui va nous permettre de donner une vision globale à cette problématique notamment dans l'étape confirmatoire. L'analyse du contenu des entretiens effectués lors de la phase exploratoire nous a permis de confirmer nos construits théoriques ainsi que l'identification de certains facteurs pertinents, traitant l'approche processus, ayant déjà fait l'objet d'un développement dans la littérature. Nous rapportons dans ce qui suit, les résultats trouvés.

\section{Thème 1: Comprendre le contexte stratégique de déclenchement d'une démarche d'approche processus}

Dans cette 1ère thématique, les répondants ont précisé que l'approche processus est une démarche qui fait partie du système de management de la qualité.

Extraits de verbatim qui illustrent notre propos :

"Pour mettre en place un système de management intégré Qualité Sécurité Environnement (QSE), l'approche processus est obligatoire. La première chose que l'auditeur externe vérifie c'est l'approche processus et son déploiement. Il vérifie aussi la maturité de l'approche processus cette opération se fait processus par processus (Rép-5) ».

"Toutes les normes relatives au Système de Management de la Qualité prévoient l'approche processus (Rép-13)».

"Ça fait partie des sept principes de management de la qualité (Exp-3) » L'aval de la direction générale reste décisif dans la mise en place de l'approche processus, car il constitue la garantie de l'adhésion et l'implication de tout le monde dans le projet : 
«Parmi les exigences de la norme ISO 9001 c'est l'engagement de la direction. L'implication du personnel ne suffit pas, il faut l'engagement du top management (Rép-3)».

«C'est très important. Dans l'étape du diagnostic, on doit détecter la tendance générale de la Direction Générale (DG). On doit savoir est ce qu'il y a vraiment volonté de mise en place d'un vraie (SMQ) ou pas. Sinon, ils seront certifiés mais après ils vont tomber dans des problèmes (Exp-2)». «Un état des lieux de l'environnement stratégique de l'entreprise est souvent dressé par les cabinets afin de s'assurer de la volonté des dirigeants quant à la mise en place de la démarche, car souvent, les objectifs exprimés diffèrent des ceux réels (Exp-2)».

S'agissant de la méthode d'implémentation de l'approche processus : « Le top management préfère être prudent et que le projet ne perturbe pas le fonctionnement normal de l'entreprise et ne crée pas de rupture (Rép-13) ».

Le personnel constitue un élément fondamental dans la réussite ou l'échec de l'approche processus. Il peut, s'il n'est pas impliqué, être un obstacle pour la réussite de ce projet : "L'implication n'était pas au niveau, le personnel n'était pas bien formé et sensibilisé. Il faut utiliser un langage simple avec les employés pour capter leur confiance et leur adhésion (Rép-9) $\gg$.

Enfin, et concernant le retour sur investissement de l'approche processus, les répondants confirment que cette démarche a un effet positif sur la performance de l'entreprise :

"L'organisation est devenue plus maitrisée, il s'agit d'un retour sur investissement qualitatif et pas quantitatif, la communication est devenue plus fluide, possibilité de détection des non conformités, baisse des coûts de la non qualité (Rép-4)».

"Il y a un retour sur investissement, mais pas en termes de chiffre d'affaire mais en termes de manque à gagner, parce qu'avec la mise en place de l'approche processus il y a des règles de contrôle, minimisation des coûts de non qualité et des pièces non conformes. Donc il y aura un minimum de respect et l'entreprise va gagner en termes de satisfaction client, car le client va avoir de la confiance dans l'entreprise, et par conséquent, il va acheter plus. L'entreprise va gagner dans la continuité c'est-à-dire la pérennisation du client (Rép-6)».

« ... une maturité accrue de l'approche processus, performance au niveau de la qualité : diminution des réclamations clients. Chez nous la réclamation client égale argent. Signature de nouveaux contrats (Rép-5) ».

"Les opérations sans valeur ajoutée une fois éliminées ils font gagner de l'argent. Réduire les pertes sèches (Rép-11)».

«La pérennisation du client car lorsque le client voit qu'on adopte une approche processus et que les processus sont mis en place il aura de la 
confiance en nous. On aura une reconduction d'une autre mission, les missions se passent bien qu'il faut avec une démarche bien ficelée. L'argent c'est la notoriété sur le marché (Exp-1)».

Les répondants confirment tous la valeur ajoutée de la démarche et son impact positif sur la performance de l'entreprise. Ils reconnaissent un retour sur investissement considérable et une capacité concurrentielle énorme si la démarche est implémentée dans les règles de l'art et selon les bonnes pratiques.

\section{Thème 2 : Comprendre les déterminants de l'approche processus}

Nous présentons ci-après les résultats de l'analyse des discours sur les facteurs clés de succès de l'approche processus issus du modèle (BPMM) :

L'alignement stratégique : les répondants précisent que l'adoption d'une approche processus est une décision de la direction générale permettant la réalisation des objectifs stratégiques de la manière la plus efficace possible.

«Il y a une logique est que pour bâtir un objectif stratégique je dois commencer du bas (logique Bottom-up) sinon l'objectif va relever de la théorie. Dans l'ISO 9001, on parle de la nécessité de comprendre le contexte et identifier les risques les opportunités (Rép-13)».

«En principe, l'objectif doit être défini en collaboration avec le pilote processus (Rép-3)».

"Les objectifs stratégiques doivent être déclinés en objectifs opérationnels dont la réalisation est confiée aux processus (Rép-5)».

"Il a été intégré dans la version 2015 de l'ISO 9001, le chapitre 4 stipule que l'entreprise doit démontrer l'impact de la démarche qualité et de l'approche processus sur les objectifs business de l'entreprise. Lors de la conception des processus, on doit penser stratégie. En fixant des objectifs pour les processus, il faut toujours établir le lien avec la stratégie (Exp-2)».

Gouvernance : L'Exp-2 définit la gouvernance comme «monitoring, management, rôles, autorités, pour chaque processus ». D'autres propos sont formulés par les répondants sur ce sujet :

«...il y a un chapitre (ch. 6) qui parle des fonctions et autorité et gouvernance, pour chaque pilote de processus il faut déterminer les fonctions, les autorités, les objectifs (Rép-5)».

«Il s'agit d'un élément important dans l'approche processus puisqu'il concerne les organes qui vont prendre en charge les processus de par ses responsabilités de la performance et de l'amélioration de ces processus ... Ce sont les pilotes des processus, ... dont la mission est de veiller à ce que les processus fonctionnent conformément aux objectifs qui leurs sont fixés en suivant leurs performance grâce à des indicateurs pertinents (Rép-1)». «... Le pilote doit former, parrainer, superviser les gens. Plus, s'il y a un volet réglementé de l'activité pour laquelle je dois rendre compte de la 
performance, du dysfonctionnement tout ça c'est la responsabilité du pilote, il peut déléguer mais d'une façon contrôlée (Rép-13)».

La réussite d'une approche processus est liée à la qualité de son système de pilotage dans lequel les pilotes de processus jouent un rôle important .

Méthodes : de nombreuses méthodes sont développées afin de permettre la mise en œuvre, l'amélioration et la promotion de la performance de l'approche processus. Les propos suivants traitent ce volet :

"Dans la politique qualité et management, il y a un chapitre appelé "outils" : 5M, Ishikawa, PDCA, 5S, SMED (Rép-5 et le Rép-8)».

«Un processus quand il tourne, comment je peux le surveiller, contrôler, ce sont les méthodes de pilotage, ce sont des outils d'analyse (Exp-2)».

«Je confirme que ces outils sont importants, car ils ne sont pas théoriques, ils viennent de la réalité, ils sont développés par des industriels (Rép-11)».

Les méthodes sont donc importantes dans la mesure où elles permettent d'apporter des améliorations aux processus et aux interfaces, néanmoins, il est constaté, d'après les entretiens, qu'elles doivent être utilisées efficacement par le choix de la bonne méthode au bon moment.

Technologie de l'information : La technologie peut aider à contrôler le changement des processus, faciliter la phase de leur conception et achever leur mise en œuvre final. Extraits de verbatim qui illustrent notre propos :

"Ça devient une nécessité et pas un privilège. L'élément le plus important pour les processus c'est l'information qui nécessite de la technologie pour sa gestion. Le flux d'information est essentiel à côté des flux physiques (Rép-12) $\gg$.

«Ça peut faciliter la modélisation des processus (Rép-1)».

«C'est un facteur très important de telle sorte qu'on a créé un processus des systèmes d'information (SI) dans la société, ça facilite la communication, permet un gain de temps, manière pour uniformiser la communication entre le personnel (Rép-2)».

"Il aide beaucoup aussi, les logiciels de qualité aident beaucoup surtout dans la gestion documentaire. Elle facilite la communication, le partage, la modification, la mise à jour ... (Rép-4)».

«Un outil comme ERP est primordial dans l'industrie automobile et son absence constitue une non-conformité (Rép-3)».

«On conseille les entreprises de mettre en place un IT (information technology) pendant la mise en place d'un $S M Q$, comme ça, chaque chapitre validé il est incorporé dans le IT. Ça nous facilite la tâche dans la conception $d u(S M Q)$. On exige à ce que l'IT aille en parallèle avec le (SMQ). On devient des parties prenantes dans la mise en place du IT (Exp-2)». 
La technologie de l'information est importante pour la conception des processus et leurs fonctionnements par la suite ce qui permet une réactivité accrue et une efficacité .

Ressources humaines : La réussite ou l'échec de l'approche processus sont étroitement liés au degré de l'implication du personnel dans la démarche, comme a été souligné clairement dans les propos suivants :

"C'est un facteur important sachant que c'est le personnel qui fait fonctionner les processus et c'est lui qui peut réussir ou faire échouer une telle démarche (Rép-1)».

"C'est le cour des processus. On ne peut pas réussir une démarche qualité sans une ressources humaine ( $R H)$ qualifiée et engagée (de l'agent de sécurité jusqu'au Directeur). Il faut une motivation, une reconnaissance (Rép-4) ».

"C'est important, quelqu'un qui est motivé pour l'exercice, qui est formé sur les techniques de pilotage et qui a une certaine autorité au sein de l'organisation. Il ne faut pas parler des ressources que pour le pilote, il faut penser aussi des intervenants dans l'étape de la conception. On parle toujours dans la revue de direction de l'adéquation des ressources (Exp-2)».

«Le volet de la polyvalence du personnel était très bénéfique en termes de mobilité (Rép-1)».

«On mise sur le capital humain, je me base sur la réduction de la taille humaine car tant on grandit tant le processus devient difficile à gérer et la qualité est difficile à suivre. J'essaie au maximum d'avoir des personnes transversales (Exp-1)».

La mise en place d'une approche processus dans l'entreprise est un projet de changement d'organisation qui doit être accompagné par un changement au niveau des manières de travailler du personnel, car en fin de compte, c'est ce dernier qui va fonctionner les processus.

Culture : les répondants ont précisé qu'il est difficile de changer la culture des gens dans peu de temps pour la rendre alignée avec les exigences d'une organisation par processus. Le Rép-6 définit la culture en tant que « ... valeurs professionnelles (comme l'esprit d'équipe,) et personnelles (l'intransigeance, discipline, ponctualité... ». Les propos suivants montrent l'importance de ce volet :

«Les valeurs de l'entreprise, le sens de l'appartenance. Il faut ajouter aussi l'éducation (Rép-12)».

"La culture est quelque chose qui descend du top management, et si tu n'as pas l'adhésion des ouvriers, l'entreprise ne sera jamais certifiée. Car le personnel est parmi les parties intéressées pertinentes (PIP), et la DG doit présenter comment elle répond aux besoins de cette (PIP) (Exp-2)».

"Pour faire réussir l'approche processus on l'a adapté au contexte de l'entreprise et à nos croyances. On a joué sur la culture des chefs de projets pour changer les mentalités et certaines attitudes (Rép-5)». 
«C'est important, on n'est pas encore arrivé à adapter la norme au contexte de l'entreprise, ce qu'on fait c'est d'adapter l'entreprise à la norme. La culture musulmane doit être prise en compte dans les normes. Les gens ont peur de la responsabilité, de la sanction (Rép-4)».

"Il faut adapter les approches à la culture. Dans les grandes structures, ce sont les gens qui adhèrent à la culture de l'entreprise (Rép-11)».

"L'approche processus est dans ses débuts et on ne peut pas s'attendre, dans le court et moyen terme, à ce que le personnel adopte un langage spécifique lié à l'approche processus. Ça va prendre du temps, mais avec les formations et l'accompagnement on peut réussir ce volet. L'essentiel est que le personnel s'adapte en termes de travail et d'opérationnalité au nouveau système (Rép1)».

L'ensemble des répondants sont d'accord sur le fait que l'alignement de la culture du personnel avec les normes de l'approche processus permet de garantir la performance du processus et la bonne implémentation du système qualité SMQ.

Succès de processus : La revue de littérature suggère que les six (06) facteurs clés de succès permettent à l'entreprise de concevoir des processus performants (succès de processus) capables d'améliorer la performance globale. Ci-après les verbatims relatifs à ce concept de succès de processus. "... un processus qu'on est capable de le maitriser. Lorsque les Key Performance Indicators (KPI) d'un processus vous permettent de localiser avec précision la cause racine d'une anomalie alors le processus est performant (Rép-3)».

«Un processus qui a pu déterminer une finalité claire, et qui arrive à atteindre sa finalité au bon moment (Rép-6) ».

«Un processus performant est un processus qui est bien mis en place. Cette performance est liée à son résultat (Rép-10)».

La mesure de la performance ou de succès d'un processus est difficile à réaliser car le succès est un phénomène multidimensionnel et compliqué. Un ensemble de caractéristiques du succès de processus est apparu lors des entretiens. Nous les avons classés en trois dimensions : l'efficacité et efficience, la qualité et l'agilité.

L'efficacité et l'efficience : "Si on a atteint les objectifs et on définit des actions d'améliorations et des actions correctives et des opportunités d'améliorations et on commence à penser de l'amélioration continue, on peut dire qu'on a entré dans l'efficience du processus (Rép-2)».

«...d'habitude lorsque le met en place un processus je fixe deux indicateurs, l'un d'efficacité et l'autre d'efficience qui sont liés à la qualité des livrables (Exp-2)».

Qualité : "L'important n'est pas de dire je suis hors objectif mais de connaitre pourquoi je suis hors objectif (cause racine) (Rép-3)». 
"Lorsque les KPI d'un processus vous permettent de localiser avec précision la cause racine d'une anomalie alors le processus est performant (Rép-3)». Agilité : «le processus doit être flexible pour accepter des changements. Dans l'ISO 9001 Version 2015 il y'a une Revue de Changement, c'est parmi les exigences de la norme, et dans le logigramme du processus, on doit prévoir les changements et comment y faire face (Rép-2)».

"C'est la capacité de l'entreprise à s'adapter à son contexte ... un processus agile est un processus qui est capable de changer de finalité ou de changer les outils pour atteindre la finalité. Parmi les bienfaits de la nouvelle exigence ISO 9001 V 2015, c'est l'analyse du contexte (Rép-6) ».

\section{Thème 3 : Comprendre les déterminants de la performance}

Les répondants soulignent qu'il n'y a pas de consensus sur la définition de la performance et reconnaissent le caractère multidimensionnel de ce concept. Le Rép-4 définit la performance comme «l'atteinte des résultats ou la réalisation des objectifs opérationnels (au niveau des processus) et stratégiques fixés par la $D G »$.

Les répondants sont interrogés sur les différentes dimensions de la performance qui tiennent compte de la nature complexe de l'entreprise. L'analyse des discours nous a permis de comprendre la tendance des réponses qui convergent vers le modèle de mesure de la performance de Kaplan et Norton (1992) : Balanced Score Card (BSC).

Nous avons classé les propos des répondants en suivant les axes du (BSC) de Kaplan et Norton. Les résultats sont les suivants:

Vision client : "La performance est multicritère, mais la DG a des priorités. L'entreprise doit être performante financièrement, dans le domaine des $R H$, de capter les bons éléments et de les retenir, pérenniser les clients ... (Rép14)».

«Les parties prenantes sont nombreuses et on doit choisir celles pertinentes et définir leurs exigences et les actions à faires pour répondre à ces exigences et les suivre et de s'assurer de leur satisfaction. Les clients sont la Partie Intéressée Pertinente PIP numéro 1 (Rép-2)».

La pérennité de l'entreprise ne peut pas être assurée sans satisfaction du client. Vision financière : "le premier objectif d'une société est de réaliser des performances financières et que le fait d'améliorer les autres performances n'est que pour réaliser des bénéfices supplémentaires (Rép-1)».

«Pour moi, je peux résumer la performance à une performance économique. La santé financière est très importante pour l'entreprise. Toute action qui va me permettre d'apporter de l'argent sera la bienvenue (Rép-7) ».

L'ensemble des répondants confirment que la performance financière est prioritaire et constitue le premier souci pour le chef d'entreprise.

Vision processus interne : «si le processus arrive à réaliser les objectifs, on peut dire que notre organisation interne est bonne (Rép-2)». 
"Ces deux axes sont liés car l'organisation interne est imposée par le client, le standard est imposé par le client et le standard configure votre processus. Des normes telles que : environnement, classification des clients, homologation et l'International Automotive Task Force (IATF) nous ont imposé une organisation par processus (Rép-8)».

Une organisation interne mal implémentée aura un coût pour l'entreprise « Une mal organisation interne = gaspillage, non maitrise, pas de performance (Rép-9)».

Vision innovation et apprentissage organisationnel : «Il faut se donner les moyens pour innover (les compétences internes), ..., un changement de contexte conduit à une adaptation qui pour la faire on doit innover. On doit donner la possibilité au personnel pour s'exprimer librement et proposer et en même temps être à l'écoute de votre environnement pour changer. Si tu suis votre client tu vas écouter ses exigences et innover pour répondre à ses besoins (Rép-6)».

«... le savoir-faire industriel acquis par le personnel va permettre d'apporter de la valeur ajoutée pour l'entreprise .... Création de bases expérimentées : étude, erreur, solutions, capitalisation (Rép-8)».

«L'apprentissage organisationnel est lié à la conduite de changement. Dans le management de projets on doit faire une sorte de capitalisation (causes d'échecs pour insérer des verrous dans les processus, facteurs de succès qui ont permis la réussite du projet : qui est ce qu'on a mis en place et qui a fait que le projet réussisse = bonnes pratiques). Ces bonnes pratiques deviennent une base d'apprentissage organisationnelle pour les ingénieurs désignés pour des projets futurs. Les bonnes pratiques peuvent être insérées dans les procédures (Exp-2)».

Les répondants sont tous d'accord sur le fait que l'innovation et l'apprentissage organisationnel sont important pour la survie de l'entreprise car ils permettent une capitalisation des savoirs et des bonnes pratiques nécessaires pour répondre aux besoins des clients.

L'analyse des discours relative à la notion de la performance a montré que cette dernière est une notion complexe et multidimensionnelle qui intègre l'ensemble des parties prenantes de l'entreprise ainsi que l'ensemble des connaissances et savoirs capitalisés permettant à l'entreprise de se distinguer par rapport à ses concurrents.

\section{Thème 4 : Comprendre la relation entre l'approche processus et la performance}

Dans le $1^{\text {er }}$ thème de l'étude qualitative, nous avons demandé aux répondants de nous clarifier le retour sur investissement de l'approche processus. Leurs réponses nous ont confirmé l'apport positif de la mise en place d'une telle démarche sur la performance de l'entreprise. Il est à rappeler 
que dans le contexte marocain, l'approche processus a été introduite grâce à la qualité dans le cadre des démarches de certification ISO. L'approche processus fait partie des exigences des normes de la qualité.

Nous présentons dans ce qui suit les résultats des entretiens concernant la relation entre l'approche processus et la performance. «L'approche processus est apparu dans la version 2000 de l'ISO, puis 2008 et 2015. L'amendement de la norme (par le comité 176) à travers un sondage mondial, chaque pays a des élites qui votent (des experts). Donc si ces votants votent pour garder cette approche, donc elle a un impact sur la performance (Rép-9)».

«L'approche processus dans la lère année n'est pas rentable. Donc si l'entreprise n'a pas une vision à long terme, elle va abandonner au bout de la lère année. C'est un mal nécessaire au début, mais après ça va générer des résultats (Rép-10)».

"On ne peut pas agir sur le chiffre d'affaire, l'approche processus permet de gagner sur les coûts...la tendance actuelle est de réduire les coûts. Réduction des gaspillages et des coûts de non qualité (Rép-12)».

«Maitrise des opérations, maitrise des coûts...elle donne à l'entreprise une visibilité pour détecter ses failles (Rép-11)».

«L'approche processus permet de mieux maitriser la production : réduit le temps d'arrêt, augmente la productivité et on gagne financièrement (Rép-10) $»$.

Nous avons ensuite interrogé les répondants sur les éléments de la performance non financière qui sont influencés par l'approche processus, les réponses sont les suivantes :

"L'approche processus est plus une organisation interne, donc l'axe processus interne est le plus concerné par cette démarche. Les transactions sont plus directes, les interfaces sont bien définies, chacun sait ce qu'il doit faire...l'entreprise pour le client est comme une boite noire, il ne sait pas ce qui se passe à l'intérieur. Il peut auditer quelque fois. Le client se sent plus à l'aise avec l'entreprise lorsqu'il sait que tout est standardisé, il a une garantie (Rép-10)».

«A mon avis la performance financière est un corolaire, l'approche processus impact la performance non financière. Tout ce qui permet de réguler, de maitriser, de travailler en bonne intelligence et de se faire une idée sur la performance qu'on va atteindre (Rép-13)».

"L'approche processus améliore la performance par la création des standards, elle facilite la création d'une norme, d'un standard... trois standards ont été ajoutés à l'IATF (création des opportunités, la responsabilité qualité et la maitrise du changement. En absence de l'approche processus ces standards ne seront pas là (Rép-8)》.

Nous avons demandé aux répondants de donner leurs avis sur la 
relation entre chaque facteur clés de succès de l'approche processus et la performance, les propos sont les suivant :

\section{Alignement stratégique - performance}

"l'engagement de la DG et la déclinaison des objectifs stratégiques en objectifs par processus aide l'entreprise à détecter d'une manière claire les processus qui sont performant et qui ont pu atteindre les objectifs assignés et les processus qui ont des problèmes et qui nécessitent des interventions pour qu'ils puissent atteindre les objectifs assignés...chaque processus doit produire un résultat d'exploitation, la somme des résultats d'exploitation des processus constitue le résultat global de l'entreprise (Rép-3)».

«Un processus qui est toujours aligné avec la stratégie (part de marché, évolution du Chiffre d'Affaire, ...) certainement on se retrouve avec une performance financière. Mais dans la conception du processus, on peut lier les processus métiers avec les besoins client et les processus de management (pilotage) avec l'aspect financier. On met dans les processus de pilotage un objectif qui est le degré d'atteinte des objectifs (on fait appel au DG qui en est le pilote). Un autre objectif c'est le résultat financier de l'entreprise (Exp-2) $\gg$.

\section{Gouvernance - performance}

«Gouvernance (implication responsabilité et autorité des pilotes) ... si tu délimite les responsabilités, tu vas gagner du temps et le temps c'est de l'argent donc moins de charge de personnel donc une performance financière (Rép-4)».

«En absence d'une gouvernance (absence de pilotes et des responsabilités) tu ne peux traiter les réclamations clients et réduire les non conformités donc le client ne sera pas satisfait (Rép-3)».

«Si les tâches et responsabilités sont identifiés, une non-conformité est facile à détecter, une réclamation du client est traitée avec efficacité (Rép-4)».

\section{Méthodes - performance}

«On ne peut être performant si on n'a pas de bonnes méthodes (Exp-1)». «Une méthode bien utilisée permet de résoudre un problème (répondre à une réclamation) et permet aussi de réduire le gaspillage (Rép-4)». «Appliquer des méthodes pour la résolution des problèmes et conflits surtout dans les interfaces est quelque chose d'important...Il faut vérifier les outils existants et quels outils pour quelle situation (Rép-2)».

\section{Technologie de l'information - performance}

«Elle aide dans la gestion documentaire et facilite la communication, le partage, la modification, la mise à jour... (Rép-4)». 
«Permet la fluidité de circulation de l'information sa disponibilité immédiate (Exp-2)». «Constitue un aide à la prise de décision (Rép-11)».

«Le processus va avoir du mal à vivre sans un système d'information (Exp1)».

\section{Ressources humaines - performance}

«Des personnes non compétentes coûte de l'argent et font perdre du temps donc, c'est l'aspect financier qui est en jeu (Rép-2)».

«Ce sont les piliers, car le processus a besoin d'acteurs ces acteurs sont des humains (Exp-1)».

«L'implication du personnel est le facteur clé qui aide l'entreprise à réaliser ses objectifs (Rép-8)».

\section{Culture - performance}

«Selon mon expérience, l'approche processus est le seul capable d'apporter de la performance à l'entreprise... La RCFI développe la culture des personnes et permet d'améliorer la performance client (Rép-8) ».

\section{Processus performant - performance}

Rappelons que les répondants ont défini un processus performant comme étant un processus qui réalise les objectifs qui lui sont fixés. Cela suppose, d'après ce qui a été soulevé dans le début de cette étude qualitative, que le processus doit être efficace, agile et de qualité. Les propos des répondants sont les suivants :

«Pour pouvoir juger si un processus est performant ou pas, je dois leur affecter des objectifs ou des résultats avec des indicateurs de mesure. Les résultats de l'ensemble des processus impactent la stratégie de l'entreprise et impactent ainsi la performance soit financière soit non financière (organisationnelle). La non-conformité ou réclamation signifient que le processus n'est pas maitrisé (Rép-4)».

«Pour parler de la performance, il faut parler de la valeur ajoutée. Ce qui donne la légitimité au processus c'est ce qui apporte aux autres (Rép-13) ». "La performance est le résultat de la valeur ajoutée déployée en vue d'atteindre des objectifs. Cette valeur ajoutée ne peut être que l'approche processus (Exp-3) », ce qui explique que le but derrière la mise en place d'une organisation par processus est la création de la valeur ajoutée et l'optimisation des ressources, choses qui sont confirmées par les répondants lors des entretiens déroulés.

Les répondants confirment l'impact positif de toutes les variables de l'approche processus réunies sur la performance de l'entreprise que ce soit financière ou non financière. 


\section{Modèle conceptuel et hypothèses de recherche}

Dans le cadre de cette étude qualitative, l'élaboration de notre modèle conceptuel a été basée sur le (BPMM) de De Bruin Tonia et Rosemann Michael, (2005), et sur le (BSC) de Kaplan et Norton, (1992). Les variables issues de ces études ont été confirmés suite à la réalisation des entretiens semidirectifs auprès des responsables de quatorze entreprises marocaines et trois experts. La discussion des résultats de l'étude exploratoire nous a permis d'élaborer la version définitive du modèle d'analyse de l'impact de l'approche processus sur la performance de l'entreprise au Maroc (cf. figure ci-après).

Figure 1. Modèle conceptuel de l'impact de l'approche processus sur la performance

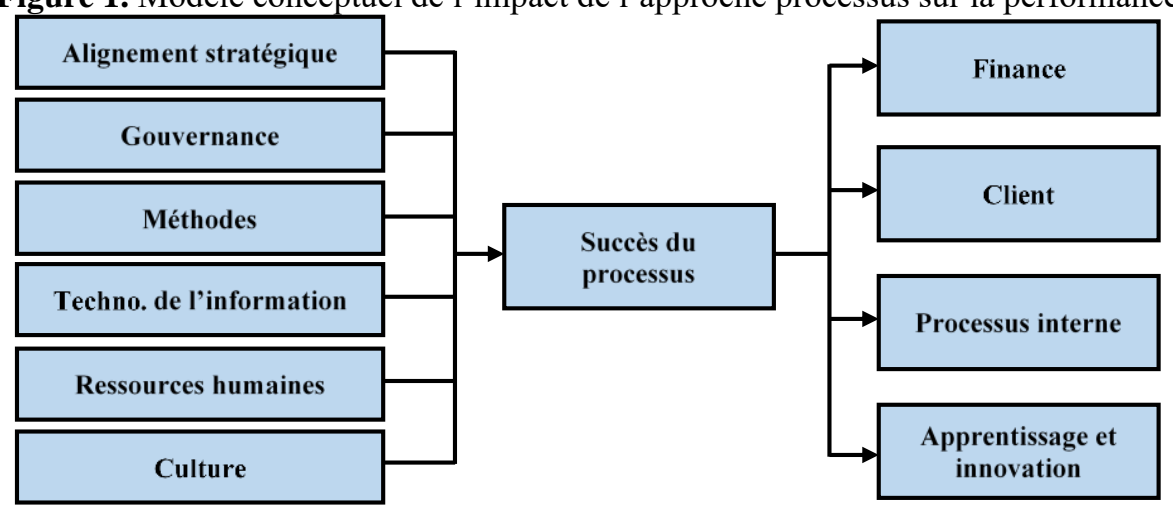

Notre modèle comporte onze variables réparties entre des variables explicatives ou variables indépendantes (6 variables), une variable médiatrice et des variables "résultats" appelées variables à expliquer ou variables dépendantes (4 variables).

D'après ce qui précède, un ensemble d'hypothèses peuvent être développées :

Tableau 6. Récapitulatif des hypothèses du modèle de recherche

\begin{tabular}{|c|c|}
\hline \multicolumn{2}{|r|}{ Hypothèses } \\
\hline H1 & $\begin{array}{l}\text { L'alignement stratégique à un effet indirect sur les différents axes de la } \\
\text { performance globale. }\end{array}$ \\
\hline H2 & $\begin{array}{l}\text { La gouvernance à un effet indirect sur les différents axes de la performance } \\
\text { globale. }\end{array}$ \\
\hline $\mathbf{H 3}$ & $\begin{array}{l}\text { Les méthodes ont un effet indirect sur les différents axes de la performance } \\
\text { globale. }\end{array}$ \\
\hline H4 & $\begin{array}{l}\text { La technologie de l'information à un effet indirect sur les différents axes de la } \\
\text { performance globale. }\end{array}$ \\
\hline H5 & $\begin{array}{l}\text { Les ressources humaines ont un effet indirect sur les différents axes de la } \\
\text { performance globale. }\end{array}$ \\
\hline H6 & La culture à un effet indirect sur les différents axes de la performance globale. \\
\hline H7 & $\begin{array}{l}\text { Le succès processus à un effet direct sur les différents axes de la performance } \\
\text { globale. }\end{array}$ \\
\hline
\end{tabular}




\section{Conclusion}

Notre étude rentre dans le cadre dans une approche qualitative exploratoire, ainsi notre méthodologie s'est reposée sur des entretiens semidirectifs que nous avons répété jusqu'à l'atteinte de la saturation théorique (Znaniecki, cité par Pires, 1997). Nous avons veillé à ce que le profil des participants soit diversifié afin d'accroître le potentiel de découverte dans notre recherche.

Toutefois, le jugement du chercheur restait présent dans le cadre d'un échantillon de choix raisonné (Thiétart R.A. et al., 2007). Au total, l'échantillon final est constitué de dix-sept personnes. A travers cette étude qualitative, nous avons pu échanger avec les répondants sur le sujet de l'influence de l'approche processus sur la performance de l'entreprise. L'analyse des discours nous permet de faire le constat que cette influence est positive dans sa globalité. Dans les entretiens, nous avons pris soins de pousser les discussions vers des détails que nous avons jugé utiles pour avoir le maximum d'informations en incitant les répondants à présenter les expériences qu'ils ont vécu dans leurs milieux professionnels.

En effet, la contribution de cette étude exploratoire concerne l'élaboration d'un modèle hypothétique de recherche à partir de deux modèles de recherche; le modèle «Business Process Management Maturity » (BPMM), de De Bruin Tonia et Rosemann Michael (2005) et le «Balanced Score Card » (BSC) de Kaplan et Norton (1992). Nous avons pu proposer notre modèle final qui décrit l'ensemble des relations contribuant à la formation de la performance induite par la mise en place de l'approche processus. La deuxième étape de notre recherche consiste à tester le modèle conceptuel proposé grâce à la méthode des équations structurelles (MES). Sur le plan théorique ce travail de recherche a pour objectif de proposer un modèle théorique au croisement de deux modèles ; «Business Process Management Maturity » et « Balanced Score Card ».

Sur le plan manageriel cette étude a permis aux responsables interviewés d'être conscients et vigilants quant à l'implémentation d'une approche processus comme déterminant d'une bonne performance. Les travaux de recherche sur l'approche processus stipulent que les entreprises ne disposant pas de processus bien définis et bien maitrisés n'arrivent pas à atteindre la performance leur permettant d'être compétitifs et leur garantissant la pérennité sur le marché.

\section{References:}

1. Allard-Poési, F. \& Maréchal, G. (1999). «Construction de l'objet de la recherche », in : Thiétart, $\mathrm{R}$. $-\mathrm{A}$. et Coll. Méthodes de recherche en management, Dunod, Paris, pp. 34-57. 
2. Adesola, S. \& Baines, T. (2005). Developing and evaluating a methodology for business process improvement. Business Process Management Journal, 11(1), 37-46.

3. Ansari, M. (1999). "Les Mesures de Perception de la Qualité Totale dans les PMI : Résultats de Recherche", Revue Internationale PME, Vol. 12, $\mathrm{N}^{\circ} .4$.

4. Bompar, A. (2010). "les facteurs influençant l'efficacité de la première relation commerciale", Thèse pour l'obtention de doctorat en Sciences de Gestion, Conservatoire National des Arts et Métiers (France).

5. Braganza, A. \& Lambert, R. (2000). Strategic Integration: Developing a Process-Governance Framework. Knowledge and Process Management, 7(3), 177-186.

6. Chareonsuk, C., \& Chansa-ngavej, C. (2010). "Intangible asset management framework: an empirical evidence". Ind. Manag. Data Syst. 110, 1094-1112.

7. Davenport, T.H. (1993). "Process Innovation-Reengineering Work through Information Technology", MIT Press, Boston-Massachusetts.

8. David R. et al. (1999). "Aide à la Décision Multicritère Pour l'Evaluation de Performance", Journal of Decision Systems, Vol. 8, $\mathrm{N}^{\circ} 3$.

9. Day, G. S. (1994). "The capabilities of market-driven organizations", Journal of Marketing, 58(4), 37-52.

10. De Bruin, T. (2009). "Business process management: theory on progression and maturity", thesis, Queensland University of Technology, Brisbane, 2009.

11. De Bruin Tonia \& Rosemann Michael (2005). "Towards a Business Process Management Maturity Model". In Bartmann, D, Rajola, F, Kallinikos, J, Avison, D, Winter, R, Ein-Dor, P, et al. (Eds.). ECIS 2005 Proceedings of the Thirteenth European Conference on Information Systems, 26-28 May 2005, Germany, Regensburg.

12. De Vaujany François-Xavier (2009). "Les grandes approches théoriques du système d'information", Hermès science / Lavoisier : Paris.

13. DeToro, I., \& McCabe, T. (1997). "How to stay flexible and elude fads", Quality Progress, 30(3), 55-60.

14. Dumas, M., van der Aalst, WM., \& ter Hofstede, AH. (2005). "Process-aware information systems: bridging people and software through process technology", Wiley, New York.

15. Elzinga, D. J., Horak, T., Lee, C.-Y., \& Bruner, C. (1995). "Business Process Management: Survey and Methodology" IEEE Transactions on Engineering Management, 42(2), 119 - 128. 
16. Evrard, Y., Pras, B., \& Roux, E. (2003). "Market : Etudes et recherches en marketing", Dunod. Paris.

17. Fitzgerald, B., \& Murphy, C. (1996). "Business process reengineering, the creation and implementation of methodology".

18. Franchini L. et al. (1997). "Conduite des Systèmes Industriels de Type PME/PMI : Problématique, Etat de l'Art et Pistes de Recherche", 2ème congrès International Fronco-québécois de Génie Industriel, ALBI.

19. Frei, F. X., Kalakota, R., Leone, A. J., \& Marx, L. M. (1999). “Process variation as a determinant of bank performance: evidence from the retail banking study", Management Science, Vol. 45 No. 9, pp. 12101220.

20. Garimella, K, Lees, M. \& Williams, B. (2008). "BPM Basics for Dummies", Software AG Special Edition, Indianapolis: Wiley Publishing, Inc.

21. Gartner (2009). Industry Research, ID Number: G00172423.

22. Garvin, D. A. (1995). "Leveraging processes for strategic advantage", Harvard Business Review, 73(5), 77-90.

23. Gautier, A. (2009). "Conduire un projet de recherche en science de gestion : la pratique de l'observatoire "in situ » et « in vivo » pour l'étude des situations de gestion", Journée des écoles doctorales d'AixMarseille, Chercher : objets, limites et contraintes, Aix-en-Province les 3 et 4 juin 2009.

24. Ginn, D., \& Barlog, R. (1994). "Reducing complexity and working with bottlenecks improves an oil refinery's engineering performance", National Productivity Review, 13(1), 63- 77.

25. Gulledge Jr, T. R. and Sommer, R. A. (2002). "Business process management: public sector implications", Business Process Management Journal, 8(4), 364-376.

26. Hammer, M. \& Champy, J. (1993). "Reengineering the Corporation. A Manifesto for Business Revolution", Jossey-Bass Inc. Publisher, London.

27. Hammer, M. (2001). "The process enterprise: An excecutive perspective", Hammer and Company, Cambridge, Massachusetts. Retrieved from http://www.hammerandco.com/.

28. Hans Brandenburg \& Jean-Pierre Wojtyna (2003). "L'approche processus, mode d'emploi ", Éditions d'Organisation Groupe Eyrolles.

29. Harrington, H. J. (1991). Business process Improvement: The Breakthrough Strategy for Total Quality, Productivity and Competitiveness, McGraw-Hill. 
30. Harmon, P. (2005). Best Practices in the Governance of BPM, viewed 18 th June 2007, http://www.bptrends.com/resources_publications.cfm.

31. Helene Bergeron (2000). "Les indicateurs de performance en contexte de PME, quel modèle appliquer ?" 21ème Congrès de l'AFC, Mai 2000, France.

32. Hill, E. J., \& Civian, J. T. (Eds.). (2008). Investigating workplace flexibility using a multi-organization database: A collaboration of academics and practitioners [Special issue]. Community, Work, and Family, 11, 1-242. doi :10.1080/13668800802172576.

33. Hinterhuber, H. H. (1995). "Business process management: the European approach", Business Change \& Re-engineering, Vol. 2 No. 4, pp : 63-73.

34. Hung, R. Y.Y. (2006). Business Process Management as Competitive Advantage: a review and empirical study. Total Quality Management, $17(1), 2-40$.

35. Ittner, C. D. \& Larcker, D. F. (1997). "The performance effects of process management techniques", Management Science, Vol. 43 No. 4, pp. 522-534.

36. ISO 9004 : 2000, Systèmes de management de la qualité - Lignes directrices pour l'amélioration des performances.

37. Jarrar, Y. F., Al-Mudimigh, A., \& Zairi, M. (2000). "ERP Implementation Critical Success Factors - The Role and Impact of Business Process Management", ICMIT 2000. Proceedings of the 2000 IEEE International Conference on Management of Innovation and Technology. 12-15 November, Singapore.

38. Kaplan, R. S., \& Norton, D. P. (1996). Translating strategy into action: the balanced scorecard. Boston, MA : Harvard Business School Press.

39. Kaplan, S. \& Norton, D. (1998). "Le Tableau de Bord Prospectif", les Editions d'Organisation, Paris.

40. Kaplan, R. S., \& Norton, D. P. (1992). "The balanced scorecardMeasures that drive performance", Harvard Business Review, 70(1), 71-79.

41. Kettinger, W.J., Teng, J.T.C., \& Guha, S. (1997). Business process change: a study of methodologies, techniques, and tools. MIS Quarterly, March, 21, 55-80.

42. Kohlbacher, M. (2009). "The Effects of Process Orientation on Customer Satisfaction, Product Quality and Time-Based Performance", 29th Annual International Conference of the Strategic Management Society, pp. 1-7.

43. Kohlbacher, M. \& Gruenwald, S. (2011). "Process orientation: conceptualization and measurement", Business Process Management 
Journal, Emerald Group Publishing Limited, Vol. 17 No. 2, pp. $267-$ 283.

44. Kohlbacher, M. \& Reijers, H.A. (2013). "The effects of processoriented organizational design on firm performance", Business Process Management Journal, Emerald Group Publishing Limited, Vol. 19 No. 2, pp. 245-262.

45. Lee, R.G. \& Dale, B.G. (1998). "Business process management: a review and evaluation". Business Process Management Journal, Vol. 4 Iss: 3, pp. $214-225$.

46. Llewellyn, N. and Armistead, C. (2000). "Business process management: Exploring social capital within processes", International Journal of Service Industry Management, 11(3), 225-243.

47. McCormack, K. (1999). "The development of a measure of business process orientation and the relationship to organizational performance", BPR online learning centre.

48. McCormack, K. (2001a). "Business process orientation: Do you have it?" Quality Progress, 34(1), 51-58.

49. McCormack, K. P. \& Johnson, W.C. (2001). "Business process orientation. Gaining the e-business competitive advantage", St. Lucie Press, Boca Raton, FL.

50. McDaniel, T. (2001). Ten pillars of business process management. eAI Journal, (November).

51. Michel Cattan (2008). "Guide des processus passons à la pratique", AFNOR Editions, 2ème édition.

52. Miles, M. B. \& Huberman, A.M. (1991). "Analyse des données qualitatives : Recueil de nouvelles méthodes", De Boeck, Bruxelles.

53. Neely, A., Gregory, M., \& Platts, K. (2005). "Performance measurement system design: a literature review and research agenda", Int. J. Oper. Prod. Manag. 25, 1228-1263.

54. Pires, A. (1997). Échantillonnage et recherche qualitative: essai théorique et méthodologique. Dans J. Poupart, J.-P. Deslauriers, L.-H. Groulx, A. Laperrière. et A. P. Pirès, (Éds). La recherche qualitative; Enjeux épistémologiques et méthodologiques (pp.113-169). Québec: Gaëtan Morin, éditeur.

55. Pritchard, J., \& Armistead, C. (1999). "Business process management - lessons from European business", Business Process Management Journal 5(1), 10-32.

56. Puah K.Y., \& Tang K.H. (2000). Business Process Management, A Consolidation of BPR and TQM. Management of Innovation and Technology - Conference Proceedings, IEEE ICMIT Conference, Singapore, 110-115. 
57. Rosemann, M. \& vom Brocke, J. (2010). "The six core elements of business process management", in vom Brocke, J. and Rosemann, M. (Eds.), "Handbook on business process management. Introduction, methods and information systems", Springer, Berlin, pp. 109-124.

58. Rowe, W.G., \& Morrow, J.L. (1999). "A note on the dimensionality of the firm financial performance construct using accounting, market and subjective measures", Can. J. Adm. Sci. 16, 58-70.

59. Selmer, C. (2003). "Concevoir le Tableau de Bord: Outil de Contrôle, de Pilotage et l'Aide à la Décision", les Editions Dunod, Paris.

60. Sharma, D.S. (2005). "The association between ISO 9000 certification and financial performance", Int. J. Account. 40, 151-172.

61. Siha, S.M., \& Saad, G.H. (2008). "Business Process improvement: empirical assessment and extensions", Business Process Management Journal, 14 (6), 778-802.

62. Škrinjar, R., Bosilj-Vukšić, V., \& Indihar-Štemberger, M. (2008). "The impact of business process orientation on financial and non-financial performance", Bus. Process Manag. J. 14, 738-754.

63. Spanyi, A. (2003). Business Process Management (BPM) is a Team Sport : Play it to win! Meghan Kiffer Pr.

64. Thiétart R.A. et al. (2007). " Méthodes de recherche en management ", Edition Dunod, Paris.

65. Van der Aalst, W.M.P., ter Hofstede, A.H.M. \& Weske, M. (2003). "Business process management: A survey", International Conference on Business Process Management (BPM 2003), Berlin, Germany.

66. Wacheux, F. (1996). "Méthodes qualitatives et recherche en gestion", Economica, Paris.

67. Zaheer, A., Rehman, K.U. \& Khan, M.A. (2010). "Development and testing of a business process orientation model to improve employee and organizational performance", African Journal of Business Management, Vol. 4 No. 2, pp. 149-161.

68. Zairi, M. (1997). "Business process management: A boundaryless approach to modern competitiveness". Business Process Management Journal, 3(1), 64-80.

69. Zairi, M. \& Sinclair, D. (1995). Business process re-engineering and process management. Business Process Reengineering \& Management Journal, 1(1), 8-30. 


\section{Annexe 1}

\section{Principaux thèmes du guide d'entretien}

\begin{tabular}{|c|c|}
\hline $\begin{array}{c}\text { Thèmes des } \\
\text { entretiens }\end{array}$ & Questions nécessaires \\
\hline $\begin{array}{l}\text { Variables de la } \\
\text { performance }\end{array}$ & $\begin{array}{l}\text { Comment comprenez-vous la performance financière? } \\
\text { Comment comprenez-vous la performance non financière? } \\
\text { - Axe client } \\
\text { - Axe processus interne } \\
\text { - Axe Innovation et Apprentissage Organisationnelle }\end{array}$ \\
\hline $\begin{array}{l}\text { Variables de } \\
\text { l'approche } \\
\text { processus }\end{array}$ & $\begin{array}{l}\text { Qu'est-ce que vous pensez des variables de l'approche processus } \\
\text { suivants : } \\
\text { - Alignement stratégique } \\
\text { - Gouvernance } \\
\text { - Méthodes } \\
\text { - Technologie de l'information } \\
\text { - Ressources humaines } \\
\text { - Culture } \\
\text { - Performance du processus } \\
\text { Selon vous, ces variables peuvent-elles être considérées comme des } \\
\text { facteurs clés de succès de l'approche processus? } \\
\text { Y a-t-il d'autres variables qui peuvent contribuer à la réussite de } \\
\text { l'approche processus? }\end{array}$ \\
\hline $\begin{array}{lr}\text { Relation entre } \\
\text { variables de } \\
\text { l'approche } \\
\text { processus et } \\
\text { performance }\end{array}$ & $\begin{array}{l}\text { D'après vous, quelles influences l'alignement stratégique exercent-t- } \\
\text { elles sur la performance financière (non financière) ? Et pourquoi ?' } \\
\text { Gouvernance } \leftrightarrow \text { performance financière (non financière) } \\
\text { Méthodes } \leftrightarrow \text { performance financière (non financière) } \\
\text { Technologie de l'information } \leftrightarrow \text { performance financière (non } \\
\text { financière) } \\
\text { Ressources humaines } \leftrightarrow \text { performance financière (non financière) } \\
\text { Culture } \leftrightarrow \text { performance financière (non financière) } \\
\text { Performance du processus } \leftrightarrow \text { performance financière (non financière) }\end{array}$ \\
\hline
\end{tabular}

${ }^{1}$ Pour les variables suivantes, les questions sont posées de la même manière. 